\title{
Effects of Rheological Parameters of a Viscoplastic Fluid on Peristaltic Pumping in a Cylindrical Tube
}

\section{H. RACHID ${ }^{1}$, M. OUAZZANI TOUHAMI ${ }^{1}$}

\author{
1. Laboratory of Mechanics, Department of Physics, Faculty of Sciences Ain Chock, Hassan II University, 5366 Maarif, \\ Casablanca, Morocco \\ rhassan2@yahoo.fr \\ touazzani@hotmail.com
}

\begin{abstract}
:
In this paper, we study theoretically the peristaltic transport of a generalized four-parameter plastic fluid in a circular cylindrical tube. The present fluid model is presented for the rheological characterization of inelastic fluid foods. Long wavelength and low Reynolds number approximations are taken into account to get solution. The effects of embedded parameters on pressure rise , frictional force and especially on the mechanical efficiency have been numerically displayed and physically discussed.
\end{abstract}

Keywords: Peristaltic transport, viscoplastic fluid, four-parameter model, mechanical efficiency

\section{Introduction}

Viscoplastic materials are fluids that exhibit a yield stress: below a certain critical threshold $\tau_{0}$ in the imposed stresses, there is no deformation and the material behaves like a rigid solid, but when that yield value is exceeded, the material flows like a fluid. Practically, such flow behavior appears in many situations, including slurries and suspensions, certain polymer solutions, crystallizing lavas, muds and clays, heavy oils, avalanches, cosmetic creams, hair gel, liquid chocolate, and some pastes. Consequently, the theory of the fluid mechanics of such materials has applications in wide array of different fields, ranging from the oil, gas and chemical industries, to food processing and to geophysical fluid dynamics. The most commonly studied such fluid is the Bingham fluid, which is often uppermost in the minds of the scientists when they think of non-Newtonian fluids. This model is a twoparameter fluid (the dynamic viscosity $\mu$ and the the yield stress $\tau_{0}$ ) and is was the object of several studies as in $[1,2]$. The suitable model is the so-called HerschellBulkley and it is a true rheological behavior over a sufficiently wide range of shear rates. This three-parameter fluid ( $\mu, \tau_{0}$ and the power law index $n$ ) has been studied bay many authors as in $[3,4]$. There are other threeparameter plastic fluids namely the Casson and the Vo- cadlo (often called Robertson-Stiff model) fluids which have been analyzed in some works [5, 6]. In 1987, Ofoli, Morgan and Steffe presented a versatile generalized fourparameter viscoplastic model (OMS) that offers improved capabilities and more flexibility over existing models in the characterization and analysis of non-Newtonian fluid foods, even for large ranges of shear rates [7]. In this paper, we attempt to investigate the effects of the rheological and geometrical parameters on the peristaltic pumping phenomenon. The numerical results of the pressure rise, frictional force and of the mechanical efficiency have been physically interpreted through graphs.

\section{Formulation and analysis}

Let us consider the peristaltic transport of a four parameter plastic fluid in a tube. The flow is induced by sinusoidal wave trains propagating with constant speed $\mathrm{c}$ along the tube walls. In the fixed frame $(\bar{R}, \bar{Z})$, the dimensional equation for the tube radius for an infinite wave train is: $H=a+b \sin \frac{2 \pi}{\lambda}(\bar{Z}-c \bar{t})$.

Where $a$ is the radius of the outer tube at inlet, $b$ is the wave amplitude, $\lambda$ is the wavelength, $c$ the wave speed, and $t$ the time.

The basic equations governing the flow of an incompressible fluid are the continuity equation and the momentum equations. For the present OMS model fluid, the extra stress tensor is given by:

$$
\bar{\tau}^{m}=\bar{\tau}_{0}^{m}+\mu_{\infty} \overline{\dot{\gamma}}^{n}
$$

$\bar{\tau}_{0}$ is the yield stress, $\mu_{\infty}$ is the high-shear limiting viscosity, $\overline{\dot{\gamma}}$ is the rate of shear, $m$ and $n$ are the power indices for behavior related to the shear stress, yield stress, and shear thinning, respectively.

We notice that the flow is unsteady in the fixed frame but in the wave frame $(\bar{r}, \bar{z})$ the flow becomes steady. These frames are related by: $\quad \bar{r}=\bar{R} ; \bar{z}=\bar{Z}-c \bar{t}$; $\bar{p}(\bar{z})=\bar{P}(\bar{Z}, \bar{t}) ; \quad \bar{u}(\bar{r}, \bar{z})=\bar{U}(\bar{R}, \bar{Z}-c \bar{t}) ;$ $\bar{w}(\bar{r}, \bar{z})=\bar{W}(\bar{R}, \bar{Z}-c \bar{t})-c ; \quad q(z)=Q(Z, t)-\pi c \bar{H}^{2}$. Where $(\bar{u}, \bar{w})$ are the radial and axial components of velocity in the wave frame and $(\bar{U}, \bar{W})$ are the radial and 
axial components of velocity in the fixed frame.

The dimensionless parameters used in the problem are defined as follows:

$$
\begin{array}{r}
z=\frac{\bar{z}}{\lambda} ; r=\frac{\bar{r}}{a} ; t=\frac{c \bar{t}}{\lambda} ; u=\frac{\lambda \bar{u}}{a c} ; w=\frac{\bar{w}}{c} ; p=\frac{a^{\frac{n}{m}+1} \bar{p}}{\mu_{\infty} \lambda c^{\frac{n}{m}}} ; \\
Q=\frac{\bar{Q}}{\pi c a^{2}} ; \tau=\frac{\bar{\tau}}{\mu_{\infty}\left(\frac{c}{a}\right)^{n / m}} ; \tau_{0}=\frac{\overline{\tau_{0}}}{\mu_{\infty}\left(\frac{c}{a}\right)^{n / m}} \\
\dot{\gamma}=\frac{\overline{\dot{\gamma}}}{\left(\frac{c}{a}\right)} ; \phi=\frac{b}{a} ; \delta=\frac{a}{\lambda} ; R e=\frac{\rho a^{\frac{n}{m}}}{\mu c^{\frac{n}{m}-2}}
\end{array}
$$

where $\delta$ is the dimensionless wave number, $R e$ is the Reynolds number and $\phi$ is the occlusion with $0<\phi<$ 1. For long wave-length $(\delta<<1)$ and negligible inertia $(R e<<1)$ approximations and using these nondimensional quantities, the basic equations become:

$$
\begin{gathered}
\frac{\partial u}{\partial r}+\frac{u}{r}+\frac{\partial w}{\partial z}=0 \\
\frac{\partial p}{\partial r}=0 ;-\frac{\partial p}{\partial z}=\frac{1}{r} \frac{\partial}{\partial r}\left(r \tau_{r z}\right) \\
\tau_{r z}=\left\{\begin{array}{cl}
{\left[\tau_{0}^{m}+\left(-\frac{\partial w}{\partial r}\right)^{n}\right]^{\frac{1}{m}},} & \left|\tau_{r z}\right|>\tau_{0} \\
\dot{\gamma}=\frac{\partial w}{\partial r}=0 \quad, \quad\left|\tau_{r z}\right| \leq \tau_{0}
\end{array}\right.
\end{gathered}
$$

The dimensionless boundary conditions are given by:

$$
\frac{\partial w}{\partial r}=0 \text { at } r=r_{0} ; w=-1 \text { at } r=H
$$

\section{Solution}

Using the boundary conditions (4) we find the solutions as follows:

- In the non-plug flow region $r \geq r_{0}$ :

$$
w=-1-\frac{1}{2^{\frac{m}{n}}}\left(-\frac{\partial p}{\partial z}\right)^{\frac{m}{n}} \int_{H}^{r}\left(\eta^{m}-(\tau H)^{m}\right)^{\frac{1}{n}} d \eta
$$

- In the plug flow region $r \leq r_{0}$ :

$$
w_{p}=w(r=\tau H)
$$

with $\tau=\frac{r_{0}}{H}=\frac{\tau_{0}}{\tau_{H}}$ with $0 \leq \tau \leq 1$. The pressure gradient is given by:

$$
\frac{\partial p}{\partial z}=2 \frac{\left(Q+h^{2}-\int_{0}^{1} h^{2} d z\right)^{\frac{n}{m}}}{\left(I_{1}+I_{2}\right)^{\frac{n}{m}}}
$$

with $I_{1}=\int_{0}^{\tau h} 2 \eta \int_{h}^{\tau h}\left(\xi^{m}-(\tau h)^{m}\right)^{\frac{1}{n}} d \xi d \eta$ and $I_{2}=$ $\int_{\tau h}^{h} 2 \eta \int_{h}^{\eta}\left(\xi^{m}-(\tau h)^{m}\right)^{\frac{1}{n}} d \xi d \eta$.
We notice that if $n=m=1, \tau=0 ; n \neq 1, m=1, \tau=$ $0 ; n=m=1, \tau \neq 0 ; n \neq 1, \tau \neq 0 ; n=m=0.5, \tau \neq$ 0 ou $n=1, m \neq 1, \tau \neq 0$ we obtain the behavior of a Newtonian; power law; Bingham; Heschell-Bulkley; Casson or Vocadlo fluids.

The pressure rise $\Delta p$ and the frictional force $F$ across one wavelength, in their non-dimensional form, are given by:

$$
\Delta p=\int_{0}^{1} \frac{\partial p}{\partial z} d z \quad ; \quad F=-\int_{0}^{1} H^{2} \frac{\partial p}{\partial z} d z
$$

The mechanical efficiency is defined as the ratio between the average rate per wavelength at which work is done by the moving fluid against a pressure head and the average rate at which the walls do work on the fluid [8]. The expression of $E$ is found as follows [9, 10]:

$$
E=\frac{Q \Delta p}{\Delta p H_{(z=0)}^{2}+F}=\frac{Q}{1+\frac{F}{\Delta p}}
$$

with $H(z=0)=1$.

\section{Results and conclusion}
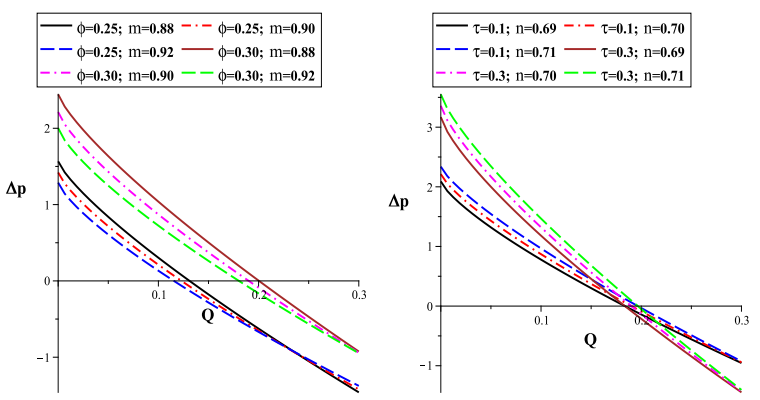

Figure 1: Pressure rise $\Delta p$ versus time-averaged flow rate $Q$.
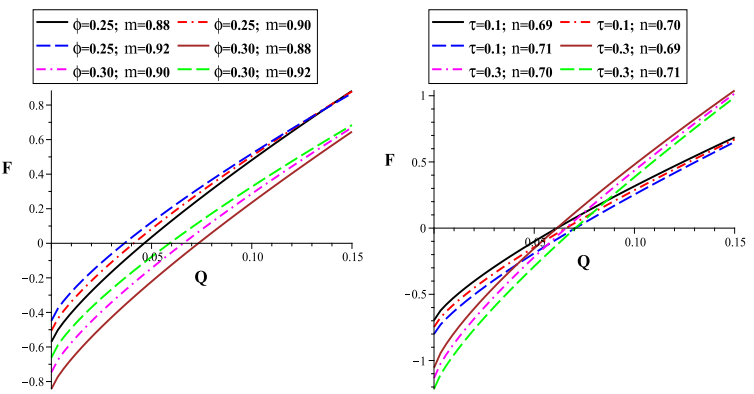

Figure 2: Frictional force $F$ versus time-averaged flow rate $Q$.
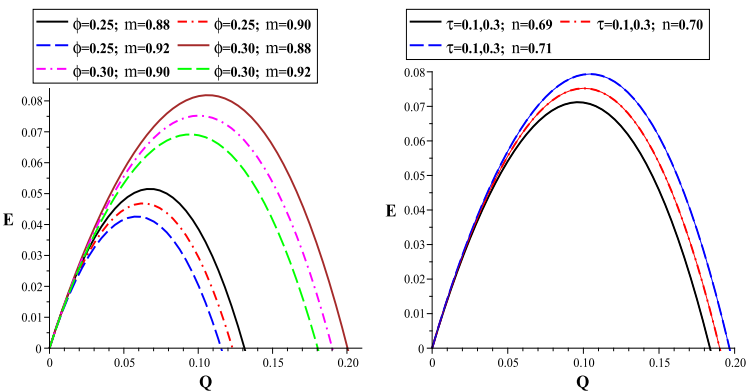

Figure 3: Mechanical efficiency $E$ versus time-averaged flow rate $Q$. 

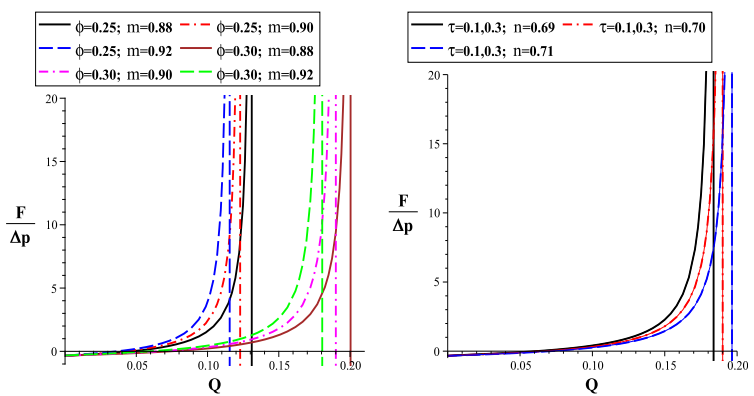

Figure 4: The ratio $\frac{F}{\Delta p}$ versus time-averaged flow rate $Q$.

In this section, we study the effect of the physical parameters of problem $(m, n, \tau$ and $\phi)$ on the pressure rise $\Delta p$, the frictional force $F$ and on the mechanical efficiency $E$ versus the time-averaged flow rate $Q$. Before we notice that for $n=m=1, \tau=0$ we find the results of Shapiro et al. [8]. In Fig. (1) we plot $\Delta p$ for different values of the power indices for behavior $m$ and $n$, yield stress threshold $\tau$ and of the amplitude ratio $\phi$. First, the figures indicate that $Q$ increases when $\Delta p$ decreases. Second, we notice that we have three regions, peristaltic pumping $(\Delta p>0$ and $Q>0)$, free-pumping $(\Delta p>0$ and $Q=0)$ and copumping ( $\Delta p>0$ and $Q<0$ ). Third, the figures show that the curves are intersecting in the co-pumping region for different values of $m, n$ and $\phi$ while they are intersecting in the free-pumping region for different values of $\tau$. Finally, we observe that the pumping $Q_{\max }$ (for $\Delta p=0$ ) increases with $n$ and $\phi$ but it decreases with $m$ while it remain the same although $\tau$ changes. Fig. (2) represents the influence of the physical parameters on the relation frictional force-averaged flow rate $F-Q$. It is found that $F$ has an opposite behavior versus $Q$ compared to $\Delta p$. Fig. (3) displays the behavior of the mechanical efficiency $E$ versus $Q$. Graphically and from Eq. (9) it is clear that $E$ increases from zero (when $Q=0$ ) to a maximum in a certain value of $Q=Q_{c}$ then it decreases to zero (when $\Delta p=0$ ). In order to explain these variations of $E$, we notice that $Q$ and the ratio frictional force/pressure rise $\frac{F}{\Delta p}$ both influence on $E$. In Fig. (4) we plot the last ratio versus $Q$ for different values of the physical parameters. The figures show that when $Q \rightarrow Q_{\max }$ we observe that $\frac{F}{\Delta p} \rightarrow \infty$ i.e, $E=0$ (see Eq. 9). For $0<Q<Q_{\max }$, the time-averaged flow rate enhances the ratio frictional fore/pressure rise because of the increase of $F$ and the decrease of $\Delta p$ with increasing $Q$ (see Figs .(1-2)). When $Q<Q_{c}$ the variation of $Q$ is dominant compared to $\frac{F}{\Delta p}$ for this reason the mechanical efficiency $E$ increases with increasing $Q$. But for $Q>Q_{c}$ the opposite remarque is observed. For the third case $Q=Q_{c}$, no one of the two quantities has a dominant effect on the mechanical efficiency i.e, $E$ becomes maximum. In addition, $m$ en- hances the ratio $\frac{F}{\Delta p}$ but $n$ and $\phi$ reduce this ration while $\tau$ has no effect on it. These last results explain the opposite effect of the physical parameters on the mechanical efficiency versus the time-averaged flow.

\section{References}

[1] Yong-Li Chen and Ke-Qin Zhu, Couette-Poiseuille flow of Bingham fluids between two porous parallel plates with slip conditions, J. Non-Newtonian Fluid Mech. 153 (2008) 1-11.

[2] P. Lakschminarayana, S. Sreenadh and G. Sucharitha The influence of slip, wall properties on the peristaltic transport of a conducting Bingham fluid with heat transfer, Procedia Engineering 127 (2015) 1087-1094.

[3] K. Vajravelu, S. Sreenadh, V. Ramesh Babu, Heat transfer analysis for the peristaltic flow of Herschell-Bulkley in a non-uniform inclined channel, Int. J. Non-Linear Mech. 40 (2005) 83-90.

[4] N. S. Akbar and A. W. Butt, Heat transfer analysis for the peristaltic flow of Herschell-Bulkley in a nonuniform inclined channel, Z. Naturforsch. 70 (2015) 23-32.

[5] S. K. Pandey and D. Tripathi, Peristaltic transport of a Casson fluid in a finite channel: Application to flows of concentrated fluids in oesophagus, Int. J. Biomath. 3 (2010) 453-472.

[6] J. J. Vocadlo and M. E. Charles, Characterization and laminar flow of fluid-like viscoplastic substances, The Can. J. Chem. Eng. 51 (1973) 116-121.

[7] R. Y. Ofoli, R. G. Morgan and J. F. Steffe, A generalized rheological model for inelastic fluid foods, J. Texture Studies 18 (1987) 213-230.

[8] A. H. Shapiro, M. Y. Jaffrin and S. Y. Weinberg, Peristaltic pumping with long wavelengths at low Reynolds number, J. fluid Mech. 37 (1969) 799-825.

[9] H. Rachid and M. T. Ouazzani, Interaction of pulsatile flow with peristatic transport of a viscoelastic fluid: case of a Maxwell fluid, Int. J. Appl. Mech. 6 (2014) 1450061.

[10] H. Rachid, M. T. Ouazzani and M. Riahi, Interaction of pulsatile flow with peristatic transport of a viscoelastic fluid: case of a Maxwell fluid, Acta Physica Pol. B 46 (2015) 2051-2066. 